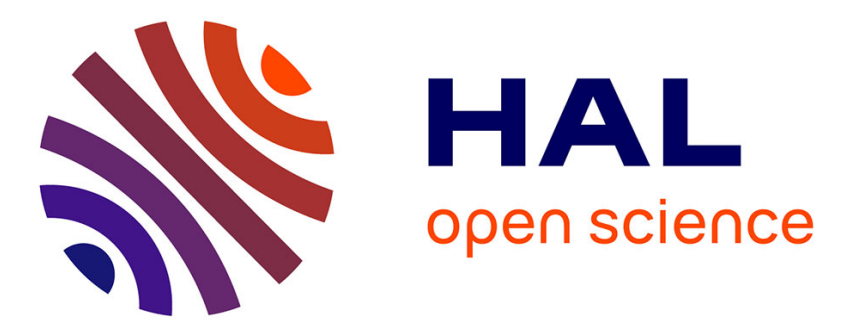

\title{
Stochastic multiscale model for HfO2-based resistive random access memories with $1 \mathrm{~T} 1 \mathrm{R}$ configuration
}

\author{
Silvana Guitarra, Laurent Raymond, Lionel Trojman
}

\section{To cite this version:}

Silvana Guitarra, Laurent Raymond, Lionel Trojman. Stochastic multiscale model for HfO2-based resistive random access memories with 1T1R configuration. Solid-State Electronics, 2021, 176, pp.107947. 10.1016/j.sse.2020.107947 . hal-03218283

\section{HAL Id: hal-03218283 \\ https://hal.science/hal-03218283}

Submitted on 5 May 2021

HAL is a multi-disciplinary open access archive for the deposit and dissemination of scientific research documents, whether they are published or not. The documents may come from teaching and research institutions in France or abroad, or from public or private research centers.
L'archive ouverte pluridisciplinaire HAL, est destinée au dépôt et à la diffusion de documents scientifiques de niveau recherche, publiés ou non, émanant des établissements d'enseignement et de recherche français ou étrangers, des laboratoires publics ou privés. 


\title{
Stochastic multiscale model for $\mathrm{HfO}_{2}$-based resistive random access memories with 1T1R configuration
}

\author{
Silvana Guitarra ${ }^{\mathrm{a}, *}$, Laurent Raymond ${ }^{\mathrm{b}}$, Lionel Trojman ${ }^{\mathrm{c}}$ \\ ${ }^{a}$ Universidad San Francisco de Quito, Colegio de Ciencias e Ingeniería, IMNE, Diego de \\ Robles s/n-Cumbayá, Quito 170901, Ecuador \\ ${ }^{b}$ Aix Marseille Univ., Université de Toulon, CNRS, CPT, Marseille, France \\ ${ }^{c}$ LISITE, ISEP, 75006 Paris, France
}

\begin{abstract}
A stochastic model for the resistive switching of ReRAM devices with 1T1R configuration is proposed in this work. This model states that the switching is caused by the changes that occur in the narrowest zone of the conductive filament due to the influence of the electric field. We work with a circuit representation of this zone where there are some breakers that can change their state according with a switching probability $\left(\mathrm{P}_{s}\right)$ that depends on the voltage drop along the breaker and the threshold voltage. This approach gives the model the stochastic behavior and allow it to generate the variability observed in most of ReRAM devices. To includes the electrical signal of the transistor, we include a series resistance. The model has been successfully validated by comparing measured and simulated IV curves of $\mathrm{HfO}_{2}$-based ReRAM devices of two different scales, $\mathrm{nm}^{2}$ and $\mu \mathrm{m}^{2}$. The flexibility and easy implementation of this resistive switching model makes it a powerful tool for the design and study of ReRAM memories.
\end{abstract}

Keywords: Resistive random access memory (ReRAM), $\mathrm{HfO}_{2}$-based memory, stochastic model, 1T1R configuration, intrinsic parameters.

\footnotetext{
* Corresponding author

Email address: sguitarra@usfq.edu.ec (Silvana Guitarra)
} 


\section{Introduction}

Resistive Random Access Memories (ReRAM) are potential candidates to be part of the next generation of non-volatile memory technology due to their promising characteristics: good cycling endurance, high speed, short switch5 ing time, low voltage operation and specially, its compatibility with CMOS industrial processes 1, 2, 3, 4. However, alike the conventional memories, the electrical response of ReRAM devices shows a stochastic behavior that can be enhanced by the type of material [5, 6]. This characteristic produces variability and makes it difficult to build an adequate predictive model.

ReRAM devices are simple MIM structure, two metallic electrodes sandwiching a transition oxide. Its memory concept is based on the resistive switching of the oxide layer by applying electrical stress. Two states, high resistive state (HRS) and low resistive state (LRS), are then possible. The transition from HRS to LRS is called SET, while the transition from LRS to HRS is called RESET. When opposite bias polarity is required for the resistive switching, the ReRAM is called bipolar.

In this work, we developed two stochastic models to understand and reliably predict the resistive switching of bipolar ReRAM devices. To do it, we examined the electrical IV curves of ReRAM cells of nine different areas. Among all possible insulator materials, we worked with Hafnium oxide $\left(\mathrm{HfO}_{2}\right)$ since it is a well-known metal-oxide material employed in the semiconductor industry [7, 8, 9. $\mathrm{HfO}_{2}$-based ReRAM devices have a filament conduction that is activated just after the electroforming process. During this step, electromigration of oxygen vacancies creates a conductive filament $(\mathrm{CF})$ inside the insulator that enables electrical conduction between the two metallic electrodes 10 . Therefore, the resistive switching is possible thanks to the local formation (set) and rupture (reset) of these filaments, driven by the applied electric field.

In the literature, models with different approaches and degrees of accuracy have been developed to simulate the resistive switching of specific ReRAM devices [11, 12, 13, 14]. However, in this work we give an unified approach which 


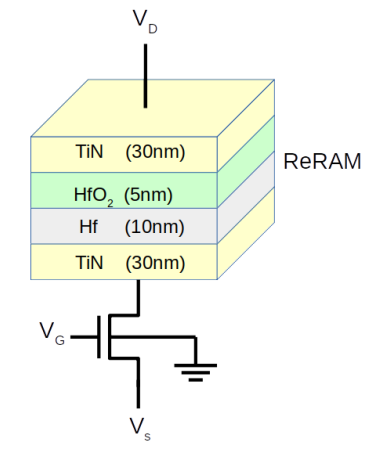

Figure 1: 1T1R ReRAM memory array

could be applied regardless of the material type, the fabrication process and the size [15. The model is based on the stochastic aspects of the resistive switching and can be calibrated with only few parameters that can be extracted directly by the analysis of IV experimental data. Further, the model include the signal of the measured element due to the 1T1R configuration. To prove the model validity we used $\mathrm{HfO}_{2}$-based $\mathrm{ReRAM}$ data.

This article is organized as follows: first, the electrical characterization of the $\mathrm{HfO}_{2}$-based ReRAM is given in Sec. 2. Then, the theoretical basis of the stochastic model is given in Sec. 3 with the corresponding comparison between experimental data and simulation results. After, the 1T1R model, that includes the electrical signal of the transistor used for measurements, is presented in Sec. 4. Finally, there is a discussion of results in Sec. 5 and the conclusions in Sec6.

\section{Electrical characterization}

We work with $\mathrm{HfO}_{2}$-based ReRAM diveces with one-transistor one-resistor

45 (1T1R) architecture (see Fig. 11. Their structure consist of $\operatorname{TiN}(30 \mathrm{~nm}) \backslash \mathrm{HfO}_{2}(5 \mathrm{~nm})$ $\backslash \mathrm{Hf}(10 \mathrm{~nm}) \backslash \operatorname{TiN}(30 \mathrm{~nm})$ stacks whose areas are: $55 \times 55 \mathrm{~nm}^{2}, 65 \times 65 \mathrm{~nm}^{2}, 75 \times 75 \mathrm{~nm}^{2}$, $85 \times 85 \mathrm{~nm}^{2}, 105 \times 105 \mathrm{~nm}^{2}, 135 \mathrm{x} 135 \mathrm{~nm}^{2}, 1 \mathrm{x} 1 \mu \mathrm{m}^{2}, 3 \mathrm{x} 3 \mu \mathrm{m}^{2}$ and $5 \times 5 \mu \mathrm{m}^{2}$. For this, the samples will be divided into two groups: nm-size samples and $\mu \mathrm{m}$ size samples. 
All devices were electroformed using positive bias voltage with a compliance current of $5 \mathrm{~mA}$. The forming voltage was between $2 \mathrm{~V}$ and $2.8 \mathrm{~V}$ in nm-size samples and between $1 \mathrm{~V}$ and $1.8 \mathrm{~V}$ in $\mu \mathrm{m}$-size. This difference could be attributed to the reduced number of intrinsic defects in fresh samples, especially in the smallest ones. Once the $\mathrm{CF}$ was formed inside the insulator, the current-voltage characteristics were measured under dc voltage sweep at room temperature. In both kind of samples, the electrical response has the typical bipolar resistive switching behavior with an asymmetric IV curve (see Fig. 22). This curves show the already known cycle to cycle variability[16, especially in nm-size samples. Further, small samples show higher resistance window $\left(\mathrm{I}_{O N} / \mathrm{I}_{O F F}\right)$ compared to big samples. This factor is essential to allow a high read margin between the two resistive states and to avoid fluctuations during operation[17].

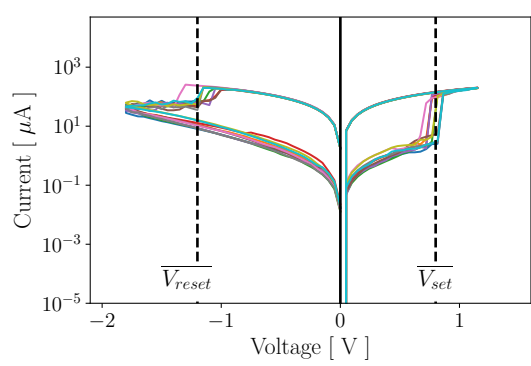

(a) $85 \times 85 \mathrm{~nm}^{2}$

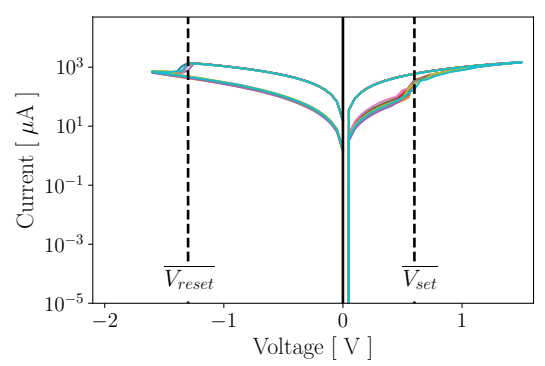

(b) $5 \times 5 \mu \mathrm{m}^{2}$

Figure 2: Current-voltage characteristics of $\mathrm{HfO}_{2}$-based ReRAM devices of $85 \times 85 \mathrm{~nm}^{2}$ and $5 \times 5 \mu \mathrm{m}^{2}$, during one experiment of ten cycles.

\subsection{Switching parameters}

From IV curves, we extracted five parameters to characterize the resistive switching. The first two are set and reset the transition voltages, $\mathrm{V}_{\text {set }}$ and $\mathrm{V}_{\text {reset }}$, that are identified by the abrupt current jump. The other three parameters are related to the conduction mechanisms.

Although the electrical response of ReRAM devices is controlled by conduction processes happening simultaneously inside the $\mathrm{CF}$ and the insulator, the current-voltage characteristic can be related to a specific model. In the case of 
$\mathrm{HfO}_{2}$-based ReRAM, the quantum point contact model (QPC) [18] is the best choice as already demonstrated by Procel et al. [19]. This model states that CF behaves as a quantum wire that allows conduction according to a potential barrier that limits the flow of electrons. In HRS the potential barrier is high and the current becomes a strongly nonlinear function of the applied voltage, while

75 in LRS the potential barrier is below the electrons energy and the conduction is ohmic. In this work, for HRS the current-voltage dependency has been defined by a hyperbolic sine function that includes the IV response under positive and negative voltages. This dependency is given by:

$$
I=I_{o} \sinh (\alpha V)
$$

where $I_{o}$ and $\alpha$ are two parameters to be determined. In LRS, the IV relationso ship is ohmic and described by:

$$
I=G V
$$

where $\mathrm{G}$ is the $\mathrm{CF}$ conductance.

All the parameters described above, $\mathrm{V}_{\text {set }}, \mathrm{V}_{\text {reset }}, G, \mathrm{I}_{o}$ and $\alpha$, were extracted independently from 150 curves for each sample. In all cases, there is a wide distribution of data that were submitted to a statistical analysis in order to determine their characteristic values and their variability. The proposed model works with the stochastic behavior of the ReRAM, so it is important to include this feature in the assessment of the electrical parameters.

In all samples, $\mathrm{V}_{\text {set }}$ and $\mathrm{V}_{\text {reset }}$ distributions can be described as unimodal, where the mean value is the most suitable measure to describe each group. 90 Normality tests were used to determine if data set in both cases is normally distributed (see insets Fig. $3 \mathrm{a}$ and $3 \mathrm{~b}$. Comparing all samples, there are two mean values of set voltage, $\mathrm{V}_{\text {set }}=0.673 \pm 0.047 \mathrm{~V}$ for $\mathrm{nm}$-size samples and $\mathrm{V}_{\text {set }}=0.480 \pm 0.007 \mathrm{~V}$ for $\mu \mathrm{m}$-size, while there is only one mean value of reset voltage $\mathrm{V}_{\text {reset }}=-1.151 \pm 0.114 \mathrm{~V}$ (Fig. $3 \mathrm{a}$ and $3 \mathrm{~b}$.

To characterize the conduction mechanism, we determined the conductance 


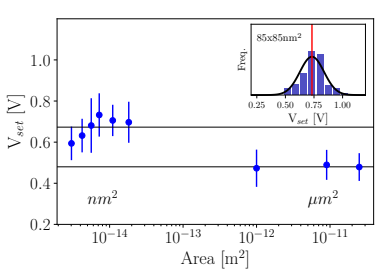

(a) $\mathrm{V}_{\text {set }}$

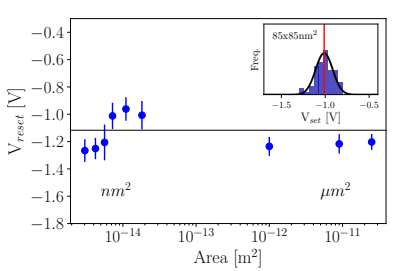

(b) $\mathrm{V}_{\text {reset }}$

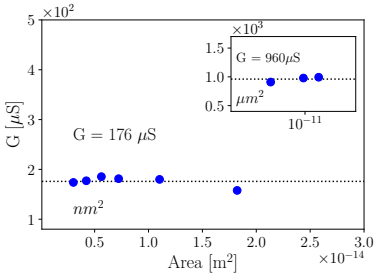

(c) $\mathrm{G}$

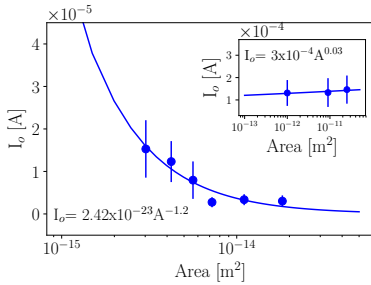

(d) $\mathrm{I}_{o}$

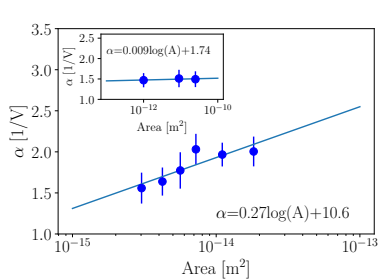

(e) $\alpha)$

Figure 3: (a) $\mathrm{V}_{\text {set }}$ and (b) $\mathrm{V}_{\text {reset }}$ as a function of the device area. Inset: Statistical distribution of $\mathrm{V}_{\text {set }}$ and (b) $\mathrm{V}_{\text {reset }}$ for the sample of $85 \mathrm{x} 85 \mathrm{~nm}^{2}$. In LRS the dominant conduction mechanism is ohmic, given by $I=G V$, where $G$ is the conductance. In HRS the conduction mechanism is tunneling, that can be studied by a relationship $I=I_{o} \sinh (\alpha V)$. The values of (a) G, (b) $\mathrm{I}_{o}$ and (c) $\alpha$ as a function of area device are presented. Insets: response of $\mu \mathrm{m}$-size samples

by the fitting of IV curves under LRS with the relationship given by Eq. 2 As one can see in Fig. $3 \mathrm{c}$, the characteristic value for nm-size samples is $\mathrm{G}=176 \pm 8.8$ $\mu \mathrm{S}$ while in $\mu \mathrm{m}$-size samples is $\mathrm{G}=960 \pm 36 \mu \mathrm{S}$. In HRS, by the fitting of the IV curves with Eq. 11 the values of $\mathrm{I}_{o}$ and $\alpha$ were determined. The large distribution of data is an evidence of the system variability in this state. Further, $\mathrm{I}_{o}$ and $\alpha$ are strongly dependent on the area (see Fig. 3d and 3e.

\subsection{Intrinsic switching parameters}

It is important to note that the conventional characterization of ReRAM devices relies on the use of one transistor (1T) to control current and protect devices from hard dielectric breakdown (see Fig. 1). This element helps to cell performance and reliability characterization, but it could also generate their own electrical signal which avoid the real characterization of the ReRAM element. 
The current controlled by the transistor is limited by an external parasitic effect that can be represented by a series resistance $\mathrm{R}_{s}[20$. Thus, to find the intrinsic switching characteristics of the ReRAM devices, the voltage drop over this $\mathrm{R}_{s}$ has been subtracted from IV curves [21. We identified this contribution by the small linear relationship after set transition in the experimental IV curves (in black) in Fig. 4, where intrinsic IV response (in red) has also been included. As expected, $\mathrm{R}_{s}$ contribution is more important in small samples.

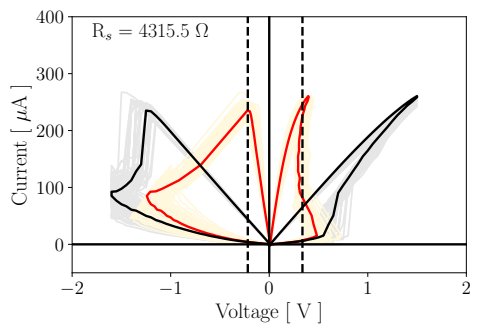

(a) $55 \times 55 \mathrm{~nm}^{2}$

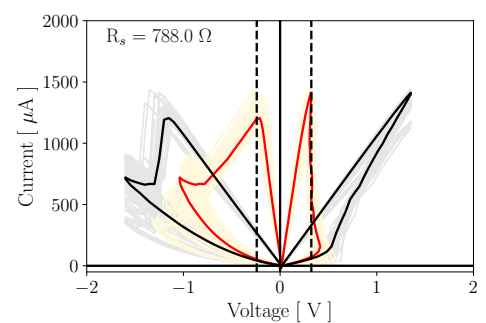

(b) $5 \times 5 \mu \mathrm{m}^{2}$

Figure 4: Experimental IV curves in grey, with mean value in black, along with the the intrinsic electrical response in yellow, with mean value in red, for the $\mathrm{HfO}_{2}$-based ReRAM.

$\mathrm{V}_{\text {set }}, \mathrm{V}_{\text {reset }}, \mathrm{G}, \mathrm{I}_{o}$ and $\alpha$, from the intrinsic IV curves. We found that $\mathrm{V}_{\text {set }}$ and $\mathrm{V}_{\text {reset }}$ trigger to a constant threshold voltage independently of the sample area (see Fig. $5 \mathrm{a}$ and $5 \mathrm{~b}$ ), as it has been previously reported by Fantini[20]. $\mathrm{I}_{o}$ and $\alpha$ were extracted by the fitting of the intrinsic curves with Eq. 11. Both of them determine intrinsic conductance $\left(\mathrm{G}_{i n t}\right)$, we extract series conductance $\left(\mathrm{G}_{s}=\frac{1}{R_{s}}\right)$ from the total conductance $\mathrm{G}$ determined before (Fig. 3c), by applying eq. 3 .

$$
\frac{1}{G_{\text {int }}}=\frac{1}{G}-\frac{1}{G_{s}}
$$

There is one characteristic value for each kind of sample (see Fig. 5c). In Fig 5 . we can compare the intrinsic parameters and those reported in section 2.1. It 


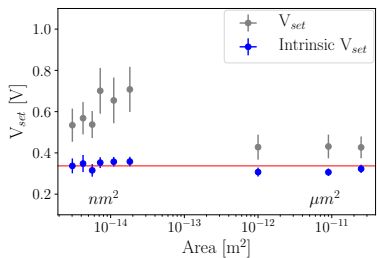

(a) Intrinsic $\mathrm{V}_{\text {set }}$

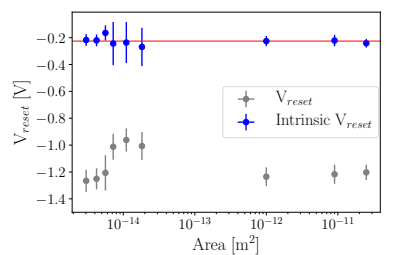

(b) Intrinsic $\mathrm{V}_{\text {reset }}$

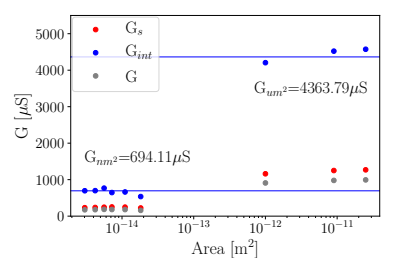

(c) Intrinsic $\mathrm{G}_{\text {int }}$

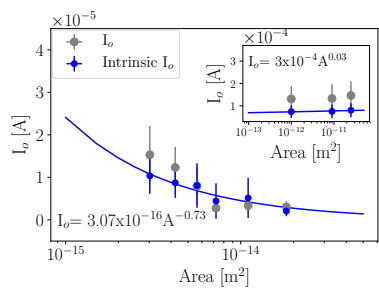

(d) Intrinsic $I_{o}$

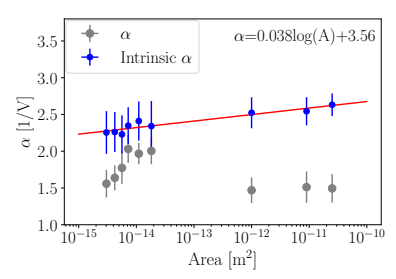

(e) Intrinsic $\alpha$

Figure 5: Intrinsic parameters of $\mathrm{HfO}_{2}$-based ReRAM as a function of the area.

\section{Stochastic model}

This paper proposes a model for the resistive switching of bipolar ReRAM device. This model works with the narrowest region of the $\mathrm{CF}$, that in the following will be called active region. As shown in Fig. 6, this region is represented this progressive switching with several vertical chains in the active region. Thus, set and reset transitions are the result of the gradual activation, or deactivation, 


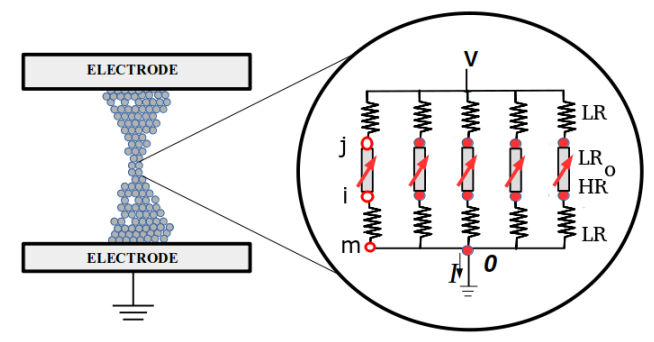

(a) Active region

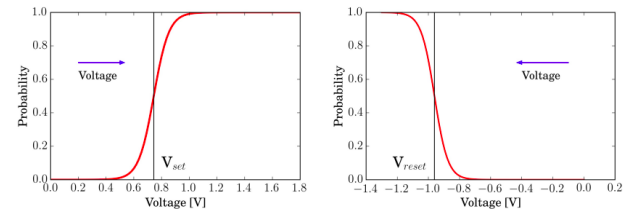

(b) Set and reset probability

Figure 6: (a) Schematic representation of the active region composed of N-chains, each one with of three electrical elements that are low resistance (LR) or high resistance (HR), and (b) Set and reset probability.

of the breakers in the active region. Nevertheless, only a stochastic process could explain the cycle to cycle variability. This characteristic has been included in our model by imposing a smooth switching probability, $\mathrm{P}_{s}$, to control the change of breaker state: $\mathrm{P}_{\text {set }}$ for set process and $\mathrm{P}_{\text {reset }}$ for reset process. A suitable mathematical expression for $\mathrm{P}_{s}$ is a sigmoid function 24, given by eq. 4 .

$$
P_{S}=\frac{1}{2}\left\{1+\tanh \left[C_{s}\left(V_{\text {link }}-V_{\text {ref }}\right)\right]\right\}
$$

where $V_{\text {ref }}$ and $C_{s}$ are two parameters that need to be determined for set and reset processes independently. $\mathrm{V}_{\text {ref }}$ is $V_{\text {set }}$ or $V_{\text {reset }}$, according to the process, and their values can be determined from experimental data. $C_{s}$ is the slope of the probability function and changes the direction and the steepness of the function. For set process the slope will be positive and open to the right, and for reset, it will be negative and open to the left (see Fig. 6b). Greater values of $\left|C_{s}\right|$ implying a very narrow transition, while the smaller ones, broader the 
node $i$ is the sum of ohmic and tunneling conduction (eq. 5), while if the breaker is LR, it is the sum of two ohmic contributions (eq. 6). This agrees with the dominant conduction mechanisms determined in section 2 for the $\mathrm{HfO}_{2}$-based ReRAM.

$$
\begin{gathered}
I_{i}=\sigma_{i m}\left(V_{i}-V_{m}\right)+I_{o} \sinh \left(\alpha\left(V_{i}-V_{j}\right)\right) \\
\left.I_{i}=\sigma_{i m}\left(V_{i}-V_{m}\right)+\sigma_{i j}\left(V_{i}-V_{j}\right)\right)
\end{gathered}
$$

In this N-chain model with two conduction mechanisms, Kirchhoff's equations result in a system of nonlinear equations that are solved by the NewtonRaphson method.

Set process is simulated following the flowchart presented in Fig. 7a. Initially, all breakers are $\mathrm{HR}$ and the external voltage $\left(\mathrm{V}_{\text {ext }}\right)$ is applied. Then, the current $(I)$ through the chains and the node voltages $\left(\mathrm{V}_{i}\right)$ are computed by solving the system of equations given by Eq. 5 and 6 . After, the value of $I$ is compared with the compliance current $\left(\mathrm{I}_{c o m p}\right)$. Only if the current is smaller than $\mathrm{I}_{\text {comp }}$, the process can continue, such as occurs in real systems to prevent dielectric breakdown of the device. Then, the drop voltage across the breaker $175\left(\mathrm{~V}_{\text {link }}\right)$ and set probability $\left(\mathrm{P}_{\text {set }}\right)$ are computed. Afterward $\left(\mathrm{P}_{\text {set }}\right)$ is compared with a computer-generated random number $p$, and only if $\mathrm{P}_{\text {set }}$ is bigger than $p$, the breaker state changes to LR, otherwise the state remains as $\mathrm{HR}$ and $\mathrm{V}_{\text {ext }}$ is increased. Once all elements in the active region are LR, the entire ReRAM is in LRS. For reset process, initially, all breakers are LR and negative bias is 

the flowchart in Fig. $7 \mathrm{~b}$

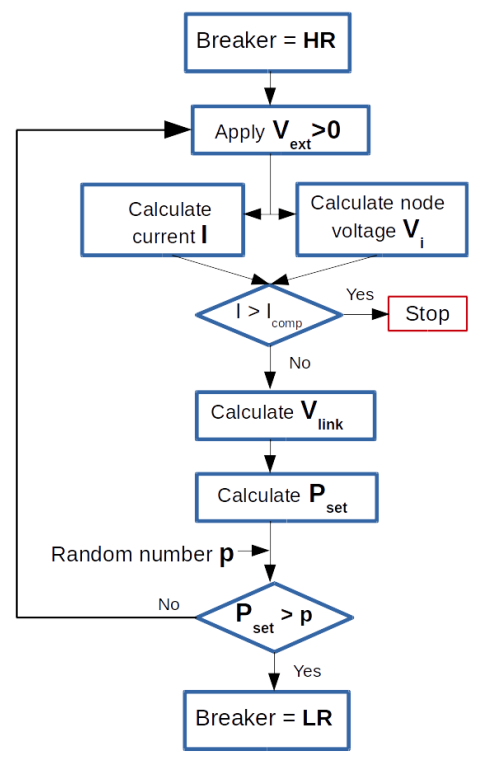

(a) Set process

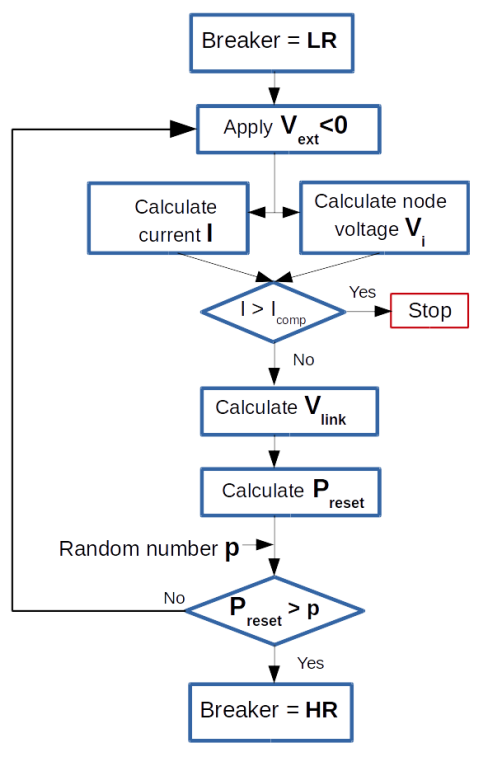

(b) Reset process

Figure 7: Flowchart used in simulation for (a)set and (b)reset processes.

\subsection{Simulation results}

First, we compared experimental data with simulation results. There is an example for one sample of each kind in Fig. 8. Several cycles have been included, in light blue experimental curves, with the mean value in blue, and in grey simulated curves with the mean value in black. For model calibration, seven parameters are required: $\mathrm{V}_{\text {set }}, \mathrm{V}_{\text {reset }}, \mathrm{G}, \mathrm{I}_{o}, \alpha, \mathrm{C}_{\text {set }}$ and $\mathrm{C}_{\text {reset }}$. They have been obtained independently for both kind of samples from the IV experimental curves (section 2). It must be noted that although both kind of samples follow similar behavior, the model was calibrated independently. As one can see inf Fig. 3. there are three parameters that are appreciably different: the conductance $\mathrm{G}$, the current $\mathrm{I}_{o}$ and the factor $\alpha$. They all characterize the $\mathrm{CF}$ conditions in LRS and HRS, thus one could suggest that even if the conduction is localized 
in the $\mathrm{CF}$, it has different size between both kinds of samples.

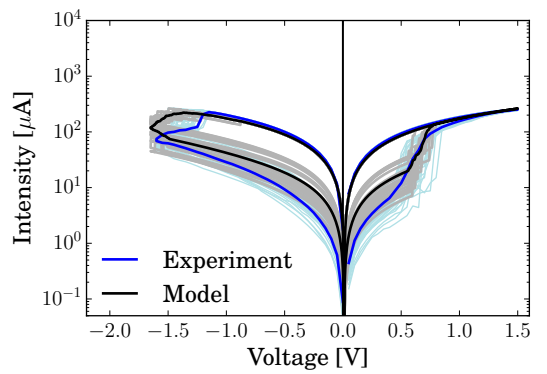

(a) $55 \times 55 \mathrm{~nm}^{2}$

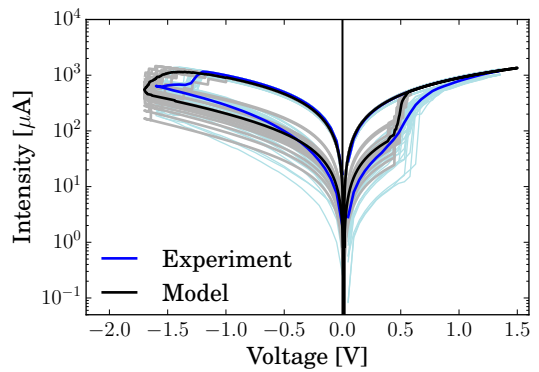

(b) $1 \mathrm{x} 1 \mu \mathrm{m}^{2}$

Figure 8: Model and experimental current-voltage response of $\mathrm{HfO}_{2} \mathrm{ReRAM}$ devices. Grey lines correspond to the model with mean value in black, while light blue lines correspond to experimental data with the mean value in blue.

As expected, the model can reproduce the gradual switching. Nevertheless, the flexibility of this model allows it to simulate an abrupt transition, like those observed in $\mathrm{Cu} / \mathrm{WOx} / \mathrm{TiN}$ ReRAM[25, if it works with one chain in the active region. Both kinds of switching, gradual or abrupt, have been reported in the literature and they are closely related to the initial CF resistance 26.

\section{1T1R Model}

Most of the models proposed in the literature for resistive switching of ReRAM devices do not consider the electrical response of the series resistance $\mathrm{R}_{s}$, associated with the 1T1R configuration used for measurement [13, 18. Here, the model proposed in section 3 has been modified to include the series resistance $\left(R_{s}\right)$. The schema of this 1T1R model is presented in Fig. 9, where there is a new node corresponding to the connection with series element, of conductance $G_{s}\left(G_{s}=1 / R_{s}\right)$. Such as in the previous case, Kirchhoff's equations at each node must to be solved according with the state of the breaker: Ohmic or TAT conduction, given by eq. 5 and 6 . This model has been implemented in a Python-based script, and calibrated with data of Table 1 that summarize all intrinsic values. The values were devided in two groups according with 
Table 1: Intrinsic parameters of $\mathrm{HfO}_{2}$-based ReRAM.

\begin{tabular}{lcr} 
Parameter & nm-size samples & $\mu$ m-size samples \\
\hline $\mathrm{V}_{\text {set }}[\mathrm{V}]$ & 0.337 & 0.337 \\
$\mathrm{~V}_{\text {reset }}[\mathrm{V}]$ & -0.226 & -0.226 \\
$\mathrm{R}_{s}[\Omega]$ & 4184.40 & 816.49 \\
$\mathrm{G}[\mu \mathrm{S}]$ & 694.11 & 4363.79 \\
$\mathrm{I}_{0}[\mu \mathrm{A}]$ & $\mathrm{I}_{o}=3.07 \times 10^{-16} \mathrm{~A}^{-0.73}$ & $\mathrm{I}_{o}=3 \times 10^{-4} \mathrm{~A}^{0.03}$ \\
$\alpha[1 / \mathrm{V}]$ & $\alpha=0.038 \log (A)+3.56$ & $\alpha=0.038 \log (A)+3.56$
\end{tabular}

sample-size.

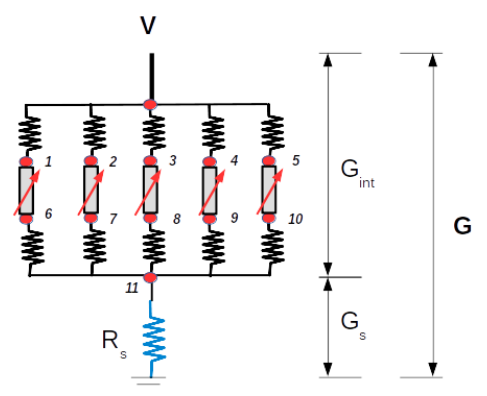

Figure 9: Schematic representation of the 1T1R Model composed of N-Chains an the series resistance $\mathrm{R}_{s}$.

Experimental data along with simulation results are presented in Fig. 10 for two samples, where grey lines correspond to the model with the mean value in 215 black, while light blue lines correspond to experimental curves with the mean value in blue. A good relationship between the experimental and the simulated curves is found in all cases.

Comparing Fig. 8 and Fig. 10, the incorporation of $\mathrm{R}_{s}$ in the model has improved the simulation, especially in the HRS. 


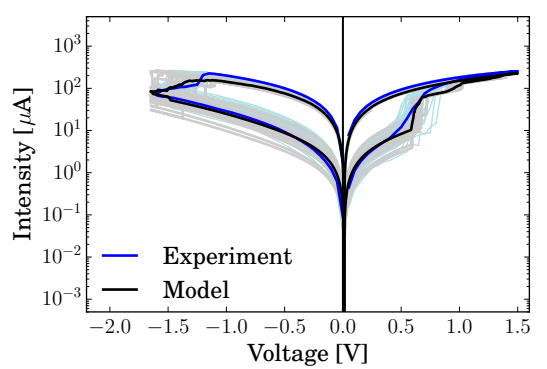

(a) $55 \times 55 \mathrm{~nm}^{2}$

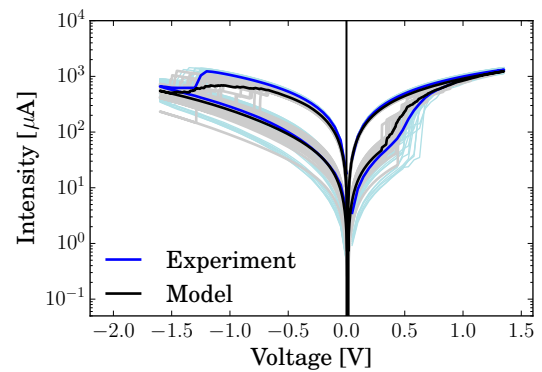

(b) $5 \times 5 \mu \mathrm{m}^{2}$

Figure 10: Model and experimental current-voltage response of $\mathrm{HfO}_{2} \mathrm{ReRAM}$ devices. Grey lines correspond to the model and light blue lines correspond to experimental curves.

\section{Discussion}

In this work, we analyze the resistive switching of ReRAM devices with only five parameters that can be extracted directly by experimental curves. Further, in order to address the intrinsic properties of the dielectric, we extracted the the signal of the transistor used in 1T1R configuration. We found that intrinsic

${ }_{225} \mathrm{~V}_{\text {set }}$ and $\mathrm{V}_{\text {reset }}$ are dependent on the dielectric material instead of the samples size (Fig. 5b and 5b) while intrinsic $\mathrm{G}, \mathrm{I}_{o}$ and $\alpha$ (see Fig. 5c, 5d and 5e) are dependent on the device area. This behavior could be related with the size of the $\mathrm{CF}$ region where the resistive switching takes place.

The variability of IV curves (Fig. 2 and 4) and, therefore, the variability of the switching parameters, is a characteristic of ReRAM devices attributed to the stochastic nature of the switching process. These feature is more evident in HRS, as has been proven by the statistical analysis of $\mathrm{I}_{o}$ and $\alpha$. This result could be interpreted by a higher distribution of energy barriers for defect migration that controls the reset process $[6$. According to QPC model, the conduction during 235 HRS includes multiple nonlinear and closely coupled parameters, such as the energy of the electrons, the potential barrier height, the shape of the barrier, a series resistance external, among others [19, 18]. In this work, we have taken the QPC arguments but we have extracted only few electrical parameters (G, $\mathrm{I}_{0}$ and $\alpha$ ) to reproduce the conduction mechanism of $\mathrm{HfO}_{2}$ ReRAMs due to the 
of two different scales, $\mathrm{nm}^{2}$-size and $\mu \mathrm{m}^{2}$-size, although the parameters used for calibration have been divided in two groups. In this sense, the impact of the area on the resistive properties has been evaluate in $\mathrm{HfO}_{2}$-based ReRAM devices.

245 vices and the active region. Our samples have a bilayer structure $\mathrm{HfO}_{2} / \mathrm{Hf}$ that allows the formation of a sub-stoichiometric region at this interface that acts as an oxygen reservoir for the resistive switching[27. According to the conductive filament mechanism, the CF could be localized inside the dielectric but or disconnected due to the effect of the electric field and provokes set and reset transition due to the movement of oxygen vacancies. This fact has been experimentally confirmed in the $\mathrm{HfO}_{2} / \mathrm{TiN}$ structure [16] and in the NiO-based system [28]. The interpretation of the resistive switching obtained from this changing to LRS occurs because of the random connection of some paths due to oxidation/reduction processes of oxygen anions and vacancies [29, 30]. This transition is produced in the model by the activation of all breakers. Contrary, when the negative voltage is applied and reaches the $\mathrm{V}_{\text {reset }}$, the rupture of the filament is caused by the filling up of the oxygen vacancies due to the drift of oxygen ions or the diffusion of oxygen ions caused by the concentration gradient of oxygen [31, 32, 33. In the model, it means the deactivation of the breakers and the transition to HRS.

In this report we propose a new modeling approach for resistive switching that incorporate the electrical signal of the measurement element (1T). This 1T1R model is consistent with the QPC model in the sense that it works with the narrow zone of the filament and also includes the series resistance external to the constriction [19, 18, 34. With this change, in the modeling, we work directly with the intrinsic characteristics of ReRAM, and improve the simulation results. 
variability reported in most of ReRAM devices. To do it, models work with a switching probability, $\mathrm{P}_{s}$, that reproduces the variability in a natural way since the algorithm includes the stochastic behavior of the process. Although a switching probability for ReRAM commutation has been proposed before in Kinetic Monte Carlo simulation[21], working with $\mathrm{P}_{s}$ at the level of circuit simulation is a new proposal. Furthermore, the calibration of this function can be done directly from the experimental evidence, such as in this work. This technique could be easily applied to other electronic devices that have random electrical behavior.

Finally, working with a switching probability to control the change of state and including the signal of the measurement element are two new good proposals in the field of memory studies that allow improving simulation results while generating variability.

\section{Conclusion}

285 We have developed two stochastic models for the resistive switching of ReRAM devices, that can simulate the IV response of bipolar cells. One of the models includes the electrical signal of the measurement element which is a new approach in the field of ReRAM simulation.

In simulation, both models work properly with devices of two different scales, $\mathrm{nm}^{2}$ and $\mu \mathrm{m}^{2}$. Although the parameters used for calibration has been divided in two groups, working with few parameter allow a quantitative description of resistive switching. Both models are able to reproduce the electrical variability observed in all ReRAM devices by using a switching probability to control the change of system state.

295

Due to the versatility of the proposed models, they could be powerful tool for simulation and studying of other tochastic devices, such as the case of the CBRAM. 


\section{References}

[1] Y. Y. Chen, L. Goux, S. Clima, B. Govoreanu, R. Degraeve, G. S.

300 TED. 2013.2241064.

[2] H. Y. Lee, Y. S. Chen, P. S. Chen, P. Y. Gu, Y. Y. Hsu, S. M. Wang, W. H. Kar, A. Fantini, G. Groeseneken, D. J. Wouters, M. Jurczak, Endurance/retention trade-off on $\mathrm{HfO}_{2} /$ Metal 1T1R bipolar RRAM, IEEE Transactions on Electron Devices 60 (3) (2013) 1114-1121. doi:10.1109/

Liu, C. H. Tsai, S. S. Sheu, P. C. Chiang, W. P. Lin, C. H. Lin, W. S. Chen, F. T. Chen, C. H. Lien, M. . Tsai, Evidence and solution of over-RESET problem for $\mathrm{HfO}_{x}$ based resistive memory with sub-ns switching speed and high endurance, in: 2010 International Electron Devices Meeting, 2010, pp. 19.7.1-19.7.4. doi:10.1109/IEDM. 2010.5703395.

[3] D. Ielmini, Resistive switching memories based on metal oxides: mechanisms, reliability and scaling, Semiconductor Science and Technology 31 (6) (2016) 063002. doi:10.1088/0268-1242/31/6/063002. URL https://doi.org/10.1088\%2F0268-1242\%2F31\%2F6\%2F063002

[4] H. Wong, H. Lee, S. Yu, Y. Chen, Y. Wu, P. Chen, B. Lee, F. Chen, M. Tsai, Metal-oxide RRAM, Proceedings of the IEEE 100 (6) (2012) 1951-1970. doi:10.1109/JPROC.2012.2190369

[5] S. Balatti, S. Ambrogio, D. Gilmer, D. Ielmini, Set variability and failure induced by complementary switching in bipolar RRAM, Electron Device Letters, IEEE 34 (2013) 861-863. doi:10.1109/LED.2013.2261451.

[6] S. Ambrogio, S. Balatti, A. Cubeta, A. Calderoni, N. Ramaswamy, D. Ielmini, Understanding switching variability and random telegraph noise in resistive RAM, in: 2013 IEEE International Electron Devices Meeting, 2013, pp. 31.5.1-31.5.4. doi:10.1109/IEDM. 2013.6724732.

[7] C. Walczyk, D. Walczyk, T. Schroeder, T. Bertaud, M. Sowinska, M. Lukosius, M. Fraschke, D. Wolansky, B. Tillack, E. Miranda, C. Wenger, Impact 
of temperature on the resistive switching behavior of embedded $\mathrm{HfO}_{2}$-based RRAM devices, IEEE Transactions on Electron Devices 58 (9) (2011) 31243131. doi:10.1109/TED.2011.2160265.

[8] S. Yu, X. Guan, H.-S. P. Wong, Conduction mechanism of TiN $/ \mathrm{HfO}_{x} / \mathrm{Pt}$ resistive switching memory: A trap-assisted-tunneling model, Applied Physics Letters 99 (6) (2011) 063507. arXiv:https://doi.org/10.1063/ 1.3624472, doi:10.1063/1.3624472 URL https : //doi .org/10.1063/1.3624472

[9] S. Privitera, G. Bersuker, S. Lombardo, C. Bongiorno, D. Gilmer, 335 - Conductive filament structure in $\mathrm{HfO}_{2}$ resistive switching memory

ㅁ devices, Solid-State Electronics 111 (2015) 161 - 165. doi:https: //doi.org/10.1016/j.sse.2015.05.044.

11 URL http://www.sciencedirect.com/science/article/pii/ S003811011500180X

[10] R. Waser, R. Dittmann, G. Staikov, K. Szot, Redox-based resistive switching memories - nanoionic mechanisms, prospects, and challenges, Advanced Materials 21 (2009) 2632-2663. doi:10.1002/adma.200900375

[11] Z. Jiang, Y. Wu, S. Yu, L. Yang, K. Song, Z. Karim, H. . P. Wong, A compact model for metal-oxide resistive random access memory with experiment verification, IEEE Transactions on Electron Devices 63 (5) (2016) 1884-1892. doi:10.1109/TED.2016.2545412.

[12] X. Guan, S. Yu, H. Wong, A spice compact model of metal oxide resistive switching memory with variations, IEEE Electron Device Letters 33 (10) (2012) 1405-1407. doi:10.1109/LED.2012.2210856.

${ }_{350}$ [13] M. Bocquet, D. Deleruyelle, H. Aziza, C. MULlER, J. Portal, T. Cabout, E. Jalaguier, Robust compact model for bipolar oxide-based resistive switching memories, IEEE Transactions on Electron Devices 61 (2014) 674681. doi:10.1109/TED.2013.2296793. 
[14] J. Noh, M. Jo, C. Kang, D. Gilmer, P. Kirsch, J. Lee, B. Lee, Development

[15] R. Degraeve, A. Fantini, N. Raghavan, L. Goux, S. Clima, Y. Y. Chen, A. Belmonte, S. Cosemans, B. Govoreanu, D. J. Wouters, P. Roussel,

[19] L. M. Prócel, L. Trojman, J. Moreno, F. Crupi, V. Maccaronio, R. Deघ graeve, L. Goux, E. Simoen, Experimental evidence of the quantum point

1 contact theory in the conduction mechanism of bipolar $\mathrm{HfO}_{2}$-based resistive random access memories, Journal of Applied Physics 114 (7) (2013) 074509. G. S. Kar, G. Groeseneken, M. Jurczak, Hourglass concept for RRAM: A dynamic and statistical device model, in: Proceedings of the 21th International Symposium on the Physical and Failure Analysis of Integrated Circuits (IPFA), 2014, pp. 245-249. doi:10.1109/IPFA.2014.6898205

[16] S. Brivio, G. Tallarida, E. Cianci, S. Spiga, Formation and disruption of conductive filaments in a $\mathrm{HfO}_{2} / \mathrm{TiN}$ structure, Nanotechnology 25 (38) (2014) 385705. doi:10.1088/0957-4484/25/38/385705. URL https : //doi.org/10.1088\%2F0957-4484\%2F25\%2F38\%2F385705

[17] S. Ambrogio, S. Balatti, V. McCaffrey, D. C. Wang, D. Ielmini, Noiseinduced resistance broadening in resistive switching memory - part ii: Array statistics, IEEE Transactions on Electron Devices 62 (11) (2015) 38123819. doi:10.1109/TED.2015.2477135.

[18] E. A. Miranda, C. Walczyk, C. Wenger, T. Schroeder, Model for the resistive switching effect in $\mathrm{HfO}_{2}$ MIM structures based on the transmission properties of narrow constrictions, IEEE Electron Device Letters 31 (6) (2010) 609-611. doi:10.1109/LED.2010.2046310

arXiv:https://doi.org/10.1063/1.4818499, doi:10.1063/1.4818499 URL https://doi.org/10.1063/1.4818499 
[20] A. Fantini, D. J. Wouters, R. Degraeve, L. Goux, L. Pantisano, G. Kar, Y. . Chen, B. Govoreanu, J. A. Kittl, L. Altimime, M. Jurczak, Intrinsic switching behavior in $\mathrm{HfO}_{2}$ RRAM by fast electrical measurements on novel 2R test structures, in: 2012 4th IEEE International Memory Workshop, 2012, pp. 1-4. doi:10.1109/IMW.2012.6213646.

[21] M. Shirasawa, M. E. Dlamini, Y. Kamakura, Kinetic monte carlo simulation for switching probability of reram, in: 2016 IEEE International Meeting

1 for Future of Electron Devices, Kansai (IMFEDK), 2016, pp. 1-1. doi: 10.1109/IMFEDK. 2016.7521667.

[22] U. Celano, L. Goux, A. Belmonte, K. Opsomer, A. Franquet, A. Schulze, C. Detavernier, O. Richard, H. Bender, M. Jurczak, W. Vandervorst,

- Three-dimensional observation of the conductive filament in nanoscaled resistive memory devices, Nano Letters 14 (5) (2014) 2401-2406, pMID: 24720425. arXiv:https://doi.org/10.1021/nl500049g, doi:10.1021/ n1500049g.

URL https://doi .org/10.1021/n1500049g

[23] S. Privitera, G. Bersuker, B. Butcher, A. Kalantarian, S. Lombardo,

घ C. Bongiorno, R. Geer, D. Gilmer, P. Kirsch, Microscopy study of the 400 conductive filament in $\mathrm{HfO}_{2}$ resistive switching memory devices, Microelectronic Engineering 109 (2013) 75 - 78, insulating Films on Semiconductors 2013. doi:https://doi.org/10.1016/j.mee.2013.03.145.

(1) URL http://wWw.sciencedirect.com/science/article/pii/ S0167931713003742

${ }_{405}[24]$ N. Kyurkchiev, S. Markov, Sigmoid Functions Some Approximation and Modelling Aspects: Some Moduli in Programming Environment MATHEMATICA, 2015.

[25] A. Masashi, A. Takahashi, Y. Ohno, A. Nakane, A. Tsurumaki-Fukuchi, Y. Takahashi, Switching operation and degradation of resistive random 
access memory composed of tungsten oxide and copper investigated using in-situ tem, Scientific reports 5 (2015) 17103. doi:10.1038/srep17103.

[26] F. Cüppers, S. Menzel, C. Bengel, A. Hardtdegen, M. von Witzleben, U. Böttger, R. Waser, S. Hoffmann-Eifert, Exploiting the switching dynamics of hfo2-based reram devices for reliable analog memristive behavior, APL Materials 7 (9) (2019) 091105. arXiv:https://doi.org/10.1063/ 1.5108654, doi:10.1063/1.5108654 URL https://doi .org/10.1063/1.5108654

[27] B. Govoreanu, G. S. Kar, Y. Chen, V. Paraschiv, S. Kubicek, A. Fantini, I. P. Radu, L. Goux, S. Clima, R. Degraeve, N. Jossart, O. Richard, T. Vandeweyer, K. Seo, P. Hendrickx, G. Pourtois, H. Bender, L. Altimime, D. J. Wouters, J. A. Kittl, M. Jurczak, 10x10nm² Hf/HfO ${ }_{x}$ crossbar resistive RAM with excellent performance, reliability and low-energy operation, in: 2011 International Electron Devices Meeting, 2011, pp. 31.6.1-31.6.4. doi:10.1109/IEDM.2011.6131652.

${ }_{425}[28]$ K. Sun-young, J. Kim, J. Choi, I. Hwang, S.-B. Hong, S.-O. Kang, B. Park, Resistive switching behaviors of nio films with controlled number of conducting filaments, Applied Physics Letters 98 (2011) 192104-192104. doi:10.1063/1.3589825,

[29] G. Bersuker, D. C. Gilmer, D. Veksler, P. Kirsch, L. Vandelli, A. Padovani, L. Larcher, K. McKenna, A. Shluger, V. Iglesias, M. Porti, M. Nafría, व Metal oxide resistive memory switching mechanism based on conductive filament properties, Journal of Applied Physics 110 (12) (2011) 124518. arXiv:https://doi.org/10.1063/1.3671565, doi:10.1063/1.3671565 URL https://doi.org/10.1063/1.3671565

${ }_{435}$ [30] S. Long, X. Lian, C. Cagli, L. Perniola, E. Miranda, M. Liu, J. Suñé, A model for the set statistics of RRAM inspired in the percolation model of oxide breakdown, IEEE Electron Device Letters 34 (8) (2013) 999-1001. doi:10.1109/LED.2013.2266332. 
[31] S. Long, L. Perniola, C. Cagli, J. Buckley, X. Lian, E. Miranda, F. Pan, M. Liu, J. Sune, Voltage and power-controlled regimes in the progressive unipolar reset transition of $\mathrm{HfO}_{x}$-based RRAM, Scientific reports 3 (2013) 2929. doi:10.1038/srep02929.

[32] U. Russo, D. Ielmini, C. Cagli, A. L. Lacaita, Self-accelerated thermal dissolution model for reset programming in unipolar resistive-switching memory (RRAM) devices, IEEE Transactions on Electron Devices 56 (2) (2009) 193-200. doi:10.1109/TED.2008.2010584.

[33] S. Yu, H. Wong, A phenomenological model for the reset mechanism of metal oxide rram, IEEE Electron Device Letters 31 (12) (2010) 1455-1457. doi:10.1109/LED.2010.2078794.

[34] S. Long, X. Lian, C. Cagli, X. Cartoixà, R. Rurali, E. Miranda, D. Jiménez, 口 L. Perniola, M. Liu, J. Suñé, Quantum-size effects in hafnium-oxide resis-

1 tive switching, Applied Physics Letters 102 (18) (2013) 183505. arXiv: https://doi.org/10.1063/1.4802265, doi:10.1063/1.4802265

URL https://doi.org/10.1063/1.4802265 